\title{
PENSIONES Y ACCIONES COMPLEMENTARIAS DE LA PROTECCION DE SEGURIDAD SOCIAL
}

\author{
Víctor Fernando Manrique López \\ Catedrático de Derecho del Trabajo \\ Universidad de Deusto
}

En memoria del gran profesor

Excmo. Sr. D. Manuel Alonso Olea

Para tratar el tema de la Seguridad Social complementaria es preciso tener en cuenta la necesidad de definir lo que se entienda por Seguridad Social.

Existen respecto de ello variaciones en el tiempo y en el espacio y hoy en el mundo industrial occidental no se entiende una Acción de Protección Social sin que la misma tenga un carácter básico y obligatorio.

En ese contexto debe entenderse lo que pueda comprender el ámbito de la llamada acción complementaria de la protección social que figura como epígrafe de esta exposición.

Viene atribuyéndose, en un primer análisis, a veces superficial, el carácter de complementario a lo que se superpone a lo básico, y se reserva el grado de lo obligatorio a lo que se opone a lo voluntario.

No es tan cierta esa oposición de términos, ya que, por un lado, en el ámbito de lo básico, cabe el diseño de un nivel complementario y en no pocas ocasiones esa complementariedad tiene el carácter de obligatoria. Así sucede con las prestaciones de Desempleo, o con el régimen reservado para los Accidentes de Trabajo y Enfermedades Profesionales en el caso de que ocurran por Falta de Medidas de Seguridad.

Por lo demás, aparecen ámbitos de la protección social en donde aun existiendo éste no cabe el concertar un aseguramiento, ni siquiera público, para el propio nivel básico, asumiendo un solo sujeto, en ese caso el empleador, el papel y la función de sujeto obligado y de sujeto responsable frente a los daños producidos por el acaecimiento de un siniestro. Este es el caso de la Incapacidad Temporal, antes denominada Incapacidad Laboral Transitoria, en la que durante un período de tiempo el empresario tiene que satisfacer a su exclusiva cuenta el 
abono de las prestaciones que para tales contingencias tiene establecidas la ley dentro del sistema público.

En tal sentido durante los cuatro primeros días no hay aseguramiento público, y en el estadio siguiente y hasta el vigésimo día el empleador se hace cargo del abono de una prestación directa, que no anticipada, sin que el asegurador público tenga que responsabilizarse de pago alguno del subsidio.

En tales casos no puede hablarse de un nivel público de aplicación directa, ni de un nivel complementario que mejore el nivel básico. No estamos ante un régimen sustitutivo.

Tampoco podemos referirnos de un modo absoluto a un nivel voluntario dentro de un régimen público, pues en éste no cabe la aparición de sujetos privados sino tan sólo en el ámbito de la gestión de intereses públicos, pero es que en el ejemplo referido empleador no es un simple gestor, sino el responsable obligado a satisfacer una cobertura que en el ámbito de las contingencias comunes le es inicialmente absolutamente ajena, ya que no encuentra encaje el fundamento de la asunción de una obligación de cobertura de asistencia por un período determinado, ni en el incumplimiento de la obligación, ni en el cumplimiento tardío o moroso, ni en la contravención de las obligaciones o de la norma.

Tampoco lo encuentra en el haber causado daño a otro interviniendo culpa o negligencia, y ello aunque se fuera a la responsabilidad delictual, contractual o incluso objetiva, pues con ello no encontraríamos respuesta adecuada para saber el motivo por el cual un empresario debe responder de las gripes de sus empleados, de los efectos de sus malas dotes como conductor y, por ende, de los accidentes de tráfico, o de sus dificultades de convivencia con el alcohol o con una vida poco ordenada médicamente.

Más difícil sería conocer con esas teorías referidas el motivo por el cual el empresario debe asegurar o dar cobertura, a través del seguro, de las enfermedades padecidas por los familiares de los trabajadores.

Es por ello, por lo que se coincide en que la fuente de estas obligaciones está en la Ley. Es ciertamente el imperativo legal dentro de un ámbito de derecho necesario, el que permite entender la atribución de aquellas responsabilidades a los empleadores.

Más si ello es así, debemos exigir al legislador que defina, con precisión, cuál es el ámbito de la Seguridad Social o protección social que queda reservada al nivel obligatorio legalmente hablando, y cuál es el del nivel complementario, reservado a la voluntariedad, y, en definitiva, a la disponibilidad.

En ese sentido nos encontramos con un legislador de diferentes épocas, las cuales obedecen a muy distintas situaciones económicas. 
El resultado es el de un panorama poco nítido y bastante difuso. Un panorama sin un diseño inicial, en el que han ido adjuntándose formulaciones que, en muchos casos, eran de respuesta a crisis económicas generales, o de la propia Seguridad Social, más o menos agudas.

Ciertamente, de un diseño inicial, en el que el objeto de la protección y del Seguro Social era el riesgo, se pasó a una etapa ulterior en donde, también con un diseño económico concreto, se pretendió, dentro de los Sistemas de Seguridad Social, proteger la contingencia.

El primero de tales proyectos tuvo una formulación económico financiera bien definida, logrando una supervivencia de alta rentabilidad económica y social de prácticamente cincuenta años. El segundo partió de posiciones diferentes y apartándose de una financiación basada en la Capitalización se acudió a un sistema de Reparto, buscando una Seguridad social expansiva.

Su solvencia tendrá también una duración aproximadamente igual, y, al cabo de otros cincuenta años, y en algunos casos sesenta años, entraría en una situación de mal diagnóstico, con muy diferentes pronósticos y en donde los especialistas no aciertan con el tratamiento, y es que ya se sabe que, como dice el dicho castizo: «un médico cura, dos dudan y tres, muerte segura».

En esa situación nos encontramos hoy, y para aproximarnos a la definición de la acción complementaria en el ámbito de la protección social, todos los autores coinciden en que se hace indispensable definir con precisión, y con carácter previo, qué es lo que sea la Seguridad Social básica.

Ya hemos visto que la Seguridad Social obligatoria no tiene por qué referirse necesariamente, según el legislador actual, a la Seguridad Social asegurada desde sujetos públicos, puesto que a las privadas se les coloca en una situación protagonista en algunos casos.

Tampoco puede entenderse que, en la Seguridad Social complementaria no intervendrán los sujetos públicos, ya que al margen de su papel intervencionista, merced a las atribuidas facultades de fiscalización, control y tutela, el legislador les permite, como aseguradores públicos, tener presencia directa en lo que inicialmente podría considerarse como el cargo más alejado de lo público, y más próximo a lo privado en el seno de la Seguridad Social.

Me refiero a la Seguridad Social negociable, pactable, o contratable en función del decisionismo y la voluntad libérrima del asegurado.

Efectivamente, se parte de un principio axiomático cual es el que la Seguridad Social básica no puede ser objeto de negociación pacto o contrato. Es esta una materia de derecho necesario que por su condición de derecho imperativo participa de la naturaleza de ius cogeos, y, 
en su seno, todo resulta indisponible. La Seguridad Social básica no se impone de acuerdo con la voluntad de los sujetos protegidos, sino absolutamente al margen de esa voluntad individual.

Esta es la tesis correcta cuando se da una precisa definición de la Seguridad social obligatoria, mas ya hemos anticipado que, en el estado actual de la situación, las cosas no responden estricta ni escrupulosamente, a los principios anteriormente reflejados.

Antes bien, sucede que, por circunstancias históricas, y, por una falta de concrección de los dos campos, el sujeto asegurador público va a tener presencia, en el ámbito de la Seguridad Social negociada, para pactar condiciones de aquella Seguridad Social básica erróneamente considerada en todos los casos como obligatoria. Este es el caso de los llamados Convenios de Seguridad Social.

Por lo demás, el legislador reservará ciertos espacios de mayor o menor dimensión para vedar la entrada al seguro, aun entendiendo que la protección otorgada por imperio de la ley forma parte de la Seguridad Social y muy particularmente de la protección social obligatoria.

Ejemplo de lo primero aparece en la posibilidad de concertar con las propias entidades gestoras la cobertura de los riesgos y contingencias básicas en función de las circunstancias personales del asegurado. Así, en el régimen de autónomos, en base a la capacidad económica del partícipe y de su edad se le facilita el que elija, lo que al profesor Barreiro en su magnífico y reciente trabajo titulado Reflexiones sobre la consideración de lo privado en la previsión social llama la protección social a la carta.

Igualmente y dentro de esa opción legislativa, que permite «contratar la Seguridad Social básica», merece destacarse las diferentes posibilidades que el legislador otorga para «desengancharse» de la Seguridad Social obligatoria, acudiendo al seguro privado, o al autoaseguramiento, para proporcionar un nivel de cobertura «equivalente» al que se ofrece desde el sistema público. Así, las empresas podrán, si cumplen los requisitos exigidos para ello, dejar de pagar «la prima» del aseguramiento público en punto a la Incapacidad Temporal o a la Asistencia Sanitaria y Maternidad, y asumir directamente las consecuencias del acaecimiento de los siniestros.

Aquí empresas como las de la Banca, Telefonía, Eléctricas y numerosas de servicios pactan con el gestor público la cobertura de un nivel básico y, por tanto teóricamente obligatorio y no negociable, alejándose del régimen de protección propio del sistema básico de la Seguridad Social.

Igualmente puede un sujeto permanecer como activo en la relación laboral, simultáneamente y como no cotizante en la relación de la Se- 
guridad Social en base a decisiones atribuibles a su sola voluntad, tal y como acaba de posibilitar el legislador para quienes no hagan caso de su crédito de pensión de jubilación y postpongan su edad de retiro.

Como se ve, tras la afirmación categórica de que en lo público no cabe la voluntariedad, pues ésta es predicable sólo de lo complementario, el legislador, partiendo de su falta de definición de situaciones, incurre en numerosas contradicciones que van a impedir marcar los terrenos de juego y amojonar las superficies de lo básico y de lo complementario.

Pero hacíamos alusión también a que en determinadas ocasiones lo que no admite el legislador es el seguro, pese a reconocer que el hecho causante debe quedar en el seno del sistema básico de la Seguridad Social.

Nos referimos consecuentemente a la responsabilidad por Falta de Medidas de Seguridad. El legislador, en el ánimo de corregir conductas antiprevencionistas o descuidadas, para con los accidentes de trabajo, impone no sólo la responsabilidad directa, sino también la prohibición del aseguramiento de esa deuda atribuible de forma directa al empresario, sin contar con que el ámbito mercantil permite a través de fórmulas elípticas dar cabida a esas situaciones aleatorias, y que, con proclamas de responsabilidad directa no se logra, frente al empresario insolvente, ninguna suerte de protección. En cualquier caso lo importante es destacar que en estos supuestos de protección añadida o complementaria-obligatoria (recargo del 30 y $50 \%$ en las prestaciones) no existe el aseguramiento necesario dentro del sistema público de la Seguridad Social.

Por otro lado, no podemos concluir esta parte de la exposición sin hacer mención a la participación directa de los sujetos públicos en la negociación de la Seguridad Social complementaria.

Ciertamente que en el nivel complementador cabe el contrato y que la fuente de la obligación son los pactos que las partes fijen libremente, más no puede olvidarse que, por un lado, la Seguridad Social puede, en ese ámbito, negociarse, como es natural, entre sujetos privados pues a ellos está reservada la iniciativa de su nacimiento y que, por la incongruencia del legislador, esa Seguridad Social complementaria puede cubrirse a virtud de los acuerdos que se alcancen con las entidades gestoras de la Seguridad Social, siendo en ocasiones obligatoria tal concertación de la llamada Seguridad Social voluntaria.

Efectivamente, tal y como ha destacado el profesor Barreiro, en el trabajo referenciado, la Seguridad Social complementaria ha podido nacer a lo largo de la historia de la Previsión en nuestro ordenamiento jurídico a través de alguna de estas tres formulaciones: 
- Mejora directa de prestaciones.

-Incremento en tipos.

- Incremento en bases.

Pues bien, los dos últimos sólo podían negociarse y concertase con la propia Seguridad Social, pese a que, al pertenecer a la misma esencia del nivel complementario, debían obedecer a criterios de estricta voluntariedad.

Añádase a ello el que la mejora directa de prestaciones, negociada o no en Convenios Colectivos, podría y puede asegurarse a través de Compañías de Seguros, Montepíos, Mutualidades, Fundaciones y Corporaciones de cualquier clase o naturaleza, al igual que con las propias Entidades Gestoras de la Seguridad Social, y esto último es lo que debe resaltarse, pues, como se ve, el propio legislador no sólo no define el campo de aplicación de lo público y obligatorio, frente a lo complementario y voluntario de la Seguridad Social, sino que, además crea una enorme confusión permitiendo y posibilitando la entrada de sujetos privados en el ámbito del nivel básico y obligatorio, para pactar las condiciones de su Seguridad Social o la de sus empleados, sino que también permite la presencia de las gestoras públicas en el ámbito voluntario, para llevar a cabo, y se supone que a virtud de transacciones, que no les son inicialmente permitidas, cuantos contratos y pactos tengan por conveniente para instrumentar el nivel de protección y el contenido del ámbito voluntario y complementario de la Seguridad Social.

Ante este estado de confusión nos hallamos, cuando el modelo entre en crisis, a partir de 1977, lo cual presupondrá la aparición de consecuencias indeseables.

Efectivamente inexistente una precisión suficiente en los conceptos de Seguridad Social básica y obligatoria, la transformación de los modelos apuntan, por un lado a la reducción de la presencia del sector público en la economía en general, por otro lado a la reducción del gasto, procurando incentivar las inversiones, y, por último, tratando de encontrar formulaciones financieras que aboguen por la sostenibilidad y solvencia de los propios regímenes de protección social especialmente en su nivel básico.

La interacción del nivel básico y el complementario es, ha sido y será una constante, con lo que, ante un diseño keynesiano de Seguridad Social, el nivel obligatorio, al ir en aumento, llevará al nivel obligatorio a un campo de mínimos. A mayor Seguridad Social obligatoria menor Seguridad Social voluntaria.

No obstante, se produce un fenómeno, a todas luces curioso cual es el siguiente. Las autoridades económicas y monetarias imponen la obli- 
gatoriedad de controlar el crecimiento de la Seguridad Social básica, más esto lo llevan a cabo, con diferente fortuna, según cual sea la idea que se posea en orden a los niveles de endeudamiento admisibles y la fijación de ese nivel de deuda máxima se sustraerá, a partir de un momento determinado, por las competencias nacidas en el ámbito de la Unión Europea, a los gobiernos de las naciones integrantes de esa comunidad económica, dejándose tal decisionismo en manos de las autoridades económicas y monetarias europeas, de forma definitiva, tras la aceptación una política de moneda única.

Pues bien, tras ello, unas y otras no se contentarán con la supervisión de lo básico, sino que pasarán a una política de control y autorizaciones para con lo complementario.

Desde ese momento las negociaciones quedarán sujetas al examen matemático y económico de tales autoridades administrativas en lo que se refiere a los contenidos de la Seguridad Social contratada, así nacida.

Los efectos de tal política de control serán inmediatos:

Por un lado, la Seguridad Social pública tratará de desvincularse de todos aquellos compromisos adquiridos por mor de su presencia en alguna de las fórmulas de la Acción Complementaria de las previsión social fijadas por la ley.

Los ejemplos son múltiples y poco aleccionadores. A veces, antisociales:

-El primer exponente de tal política de desencuentro con las obligaciones asumidas lo encontramos en la desaparición del régimen de incremento en tipos y su aseguramiento obligatorio con las propias gestoras de la Seguridad Social.

-El segundo, en el rechazo a la cobertura de lo asegurado a través de un régimen de prestaciones mejoradas por incremento en bases. Las decisiones adoptadas en ese sentido por las gestoras de la Seguridad Social en los años 90 a 93 resultan sencillamente inauditas. Plantillas enteras de empleados, como los de la empresa Valca, vieron frustradas sus legítimas expectativas en base a argumentos ciertamente peregrinos.

-El tercer rechazo lo fue para con la cobertura de las obligaciones derivadas por la concertación de coberturas nacidas, como mejora directa de prestaciones, con la finalidad de que, en virtud del cobro por la gestora de la Seguridad Social de «una prima» complementaria, ninguna de las pensiones de invalidez, viudedad u orfandad resultaran inferiores al Salario Mínimo Interprofesional. Colectivos enteros de trabajadores y beneficiarios de un sec- 
tor, como el siderometalúrgico, presenciaron hasta 1995 el lamentable espectáculo de unos gestores desentendiéndose de las obligaciones inicialmente asumidas por virtud de compromisos lícitamente adquiridos, y que, al tiempo del pago de las prestaciones, resultaba políticamente conveniente rechazar.

- A partir de ese momento se observa una acción de gestión recaudatoria, en el ámbito del Servicio Común de la Tesorería verdaderamente eficaz. Simultáneamente se observará progresivamente y de forma muy especial en los últimos años, una labor en el campo de la Afiliación del mayor rigor, lo que da como resultado, junto con el control del gasto, la aparición de un nuevo horizonte económico, nada negativo, en las finanzas de la Seguridad Social y de su tesorería.

Ahora bien, este fenómeno es coyuntural y exige que se defina con la mayor precisión posible el campo de lo básico y de lo obligatorio, frente a lo complementario y voluntario.

En caso contrario, continuaremos con una incertidumbre en el proyecto definitivo, que, a nivel nacional y comunitario, debe tener la Seguridad Social.

A modo de resumen, podemos hacer mención a tres situaciones que acreditan la urgente necesidad de clarificar los niveles:

$\left.1^{\circ}{ }^{\circ}\right)$ El afán de control y de fijación de las obligaciones y compromisos a las partes, en punto a la Seguridad Social complementaria, desde el Ministerio de Economía, con frecuentes y negativas situaciones de falta de coordinación con el Ministerio de Trabajo.

Prueba palpable de ello viene a ser la confección de proyectos de norma, y aprobación de disposiciones reglamentarias, tratando de impedir el que por vía de acuerdos, especialmente colectivos, pueda nacer un régimen de protección social complementario que no sea del gusto de los Departamentos económicos, que alcance un nivel no deseado, o que escape, en su fiscalización, a los controles fijados con sede económica en aquellos Departamentos ministeriales.

Esto se llevará a cabo ignorando el concepto, alcance y contenido de lo que venía siendo admitido como Convenio Colectivo, e incluso las nuevas normas se verán limitadas y con graves dificultades de interpretación, en tanto que desconocen el significado propio, que, en el ámbito laboral y de Seguridad Social, tienen los términos de empresario y trabajador, condición más beneficiosa o derecho adquirido.

Así se suscitó ante los Tribunales el alcance de la antigüedad en orden a la definición de los servicios pasados, como fue el caso de varias entidades de derecho público que actúan como Consorcio de Aguas. 
Igualmente, se debatió el concepto de derechos consolidados, ante la variación constante de éstos, al tiempo de fijar su valor contable y actuarial, que se modifica, según cual sea el resultado de la inversión de cada ejercicio anual, lo que, de por sí, es aleatorio y variable especialmente si no se invierte en renta fija. Así sucedió en el ámbito de numerosas empresas eléctricas.

Se debatió el término patrimonio del Fondo, a la hora de proceder a su liquidación, para su transformación, llegándose a discutir, en el ámbito de la Compañía Telefónica la posibilidad de llevar a cabo una cuantificación global del propio patrimonio al pasar de un régimen de prestación definida a otro de aportación definida.

Se discutió igualmente la eficacia erga omnes de un pacto alcanzado en el ámbito de la Seguridad Social negociado empresarialmente en la empresa Repsol, frente a quienes no aceptaran adherirse al nuevo régimen provisional sustituto del anterior.

Se planteó conflicto sobre la validez de la fijación de un plazo para adherirse a un plan nuevo con el contenido y alcance propio del mismo en relación con una importante Mutua de accidentes de tráfico.

Se sometió a pleito la posibilidad de constitución de un nuevo Plan de Pensiones a través de un convenio extraestatutario, y no por medio de otro de carácter estatutario en la empresa Iberdrola.

Se negó competencia a una Comisión negociadora de un Convenio Colectivo regular para instaurar, en su seno, Plan de Pensiones distinto al de las dos empresas que habían venido a fusionarse en el caso de $\mathrm{Hi}$ droeléctrica Española.

Se consideró que no había posibilidad de sostener dos regímenes de previsión diferenciados en el ámbito de una misma empresa, o de empresas agrupadas, incluso laboralmente hablando, como fue el caso de la Banca.

Se negaron competencias a la Comisión negociadora de un convenio como el de Iberdrola para sentar los criterios de liquidación de un Plan, con fijación de los importes correspondientes a los servicios pasados.

Y por último, se consideró necesitado de un debate judicial el determinar si son movilizables los presuntos derechos del personal de las entidades de ahorro y en concreto de una de las de Cataluña, cuando aún no habían constituido un Plan de Pensiones, en el caso de cese o baja voluntaria en la empresa, y en los diferentes supuestos de despido y de extinción del contrato de trabajo.

Numerosos pleitos, en fin, y contenciosos dispares y de una gran variedad, algunos por importes superiores a los 30.000 millones de pe- 
setas, se desarrollarán ante los Tribunales de la jurisdicción social en todas sus instancias y grados. Algunos de los pleitos referidos supusieron las existencia de más de treinta sentencias de conflicto colectivo ante la Audiencia Nacional y la Sala IV del Tribunal Supremo, amén de numerosos conflictos individuales seguidos ante los Juzgados de lo Social y los diferentes Tribunales Superiores de Justicia.

Y todo ello debido a la confusión de dos ámbitos que deben quedar deslindados por ley, y sin lo cual no podrá determinarse el nivel de cobertura alcanzable por uno y otro campo: el de la Seguridad Social básica obligatoria y el de la complementaria.

Al margen de la existencia de todo un conflicto vivo, latente y prolongado, que no beneficia al crecimiento ni a la estabilidad jurídica y financiera de los dos espacios que están llamados necesariamente a entenderse. El ejemplo máximo ha estado constituido por los pleitos planteados en el proceso de debate respecto de estos conceptos en la Caixa y en Firestone.

En este último de lo que se resuelva pende nada menos que la permanencia de la empresa en España y de la presencia igualmente del inversor japonés en nuestra economía de sector.

Tras los debates judiciales aparecen, eso sí, muy tardíamente, las reformas legislativas para incorporar al ordenamiento jurídico el resultado de aquellos contenciosos.

En todo caso parece quizás más apropiado resolver las dudas a través de la acción legislativa y no de los pronunciamientos judiciales.

Estas novedades normativas sintéticamente podrían reducirse de forma esquemática a los siguientes cambios, hoy ya en vigor, y que no se habían basado en sentencias jurisdiccionales, de lentísima gestación si el legislador hubiera clasificado la situación desde un primer momento, basándose en los propios conceptos inequívocos que le ofrecía el Derecho del Trabajo: Convenio, Pacto Extraestatutario, expectativa de derecho, etc.

Las novedades legislativas en el ámbito de la Previsión Social Complementaria introducidas por la Ley 24/2001 son las siguientes:

I. Principales novedades relativas a aspectos regulatorios: Planes de pensiones del sistema de empleo.

1. En Convenio Colectivo se podrá prever la incorporación directa de los empleados al Plan de Pensiones, salvo que éstos declaren su negativa a ello.

2. Se permite la adhesión inmediata de los empleados al Plan de Pensiones, siendo posible desde el ingreso del empleado en la plantilla del promotor. 
3. La condición de partícipe podrá extenderse a los socios trabajadores y de trabajo en los Planes de Pensiones de empleo promovido en el ámbito de sociedades cooperativas y laborales.

4. En la Comisión de Control, la representación del promotor será, con con carácter general, paritaria (50\%).

I.2. La representación de las partes integrantes en la Comisión de Control, se articula de la siguiente manera:

1. Si el Plan es de aportación definida para la contingencia de jubilación las decisiones de inversión requerirán, al menos, el voto favorable de la mitad de los representantes de los partícipes.

2. Si el Plan es de prestación definida o mixto, las decisiones que afecten al coste económico asumido por la empresa de las prestaciones definidas requerirá, al menos, el voto favorable de la mitad de los representantes del promotor.

I.3. Aparece una norma transitoria de tres años para la aplicación del nuevo principio de paridad.

I.4. Por lo demás, los Planes de Pensiones del sistema de empleo sólo podrán integrarse en fondos de pensiones que integren plantes de pensiones de dicho sistema.

I.5. Se admiten los Planes de pensiones de promoción conjunta:

1. Se permite la promoción de Planes de Pensiones de promoción conjunta de la modalidad de aportación definida, mixta o de prestación definida.

2. Las empresas promotoras de varios Planes de empleo, que, por operaciones societarias, se encuentren con varios Planes de Pensiones deberá integrarlos en un único Plan en un plazo de doce meses.

B. Las Modificaciones Generales pueden en consecuencia resumirse del siguiente modo:

1. Las entidades gestoras de Fondos de pensiones podrán contratar la gestión de las inversiones de los Fondos de pensiones que administran con terceras entidades.

2. Las entidades depositarias de Fondos de pensiones podrán confiar en un tercero la gestión, administración y depósito de los mismos, sin que, por ello, pierdan su responsabilidad de custodia.

3. Cuando la estructura y organización del mercado de Planes de pensiones lo permita, se desarrollará la contratación por vía electrónica de Planes de pensiones. 
Vistas estas reformas llevadas a cabo a impulsos de la existencia de numerosos pleitos puede decirse que, la confusión puede, por lo demás, agravarse si en el ámbito de la Unión Europea no se exige la especificación nítida de lo que deben ocupar cada uno de los dos campos, y si no se parte de la aceptación de unos conceptos unívocos y de general aceptación, al tiempo de alumbrar los acuerdos, que, en su seno, se están gestando, a saber: por un lado el proyecto de Directiva del Parlamento Europeo y del Consejo relativa a las actividades y a la supervisión de los Fondos de Pensiones de Empleo, que, bajo el número de expediente interinstitucional 2000/0260 se está debatiendo y que ha sido redactada en su última versión con fecha 3 de octubre y 8 de octubre del año 2002, la cual fue precedida de las consultas correspondientes a los interlocutores sociales, sobre la posible aprobación de una norma comunitaria, acerca de la movilización y traspaso a cualquier ámbito nacional de otro país de la Unión Europea de los derechos sobre pensiones complementarias, lo que finalizó el 2 de julio de 2002, y se recogió en un texto de dicha fecha por el Consejo de la Unión.

Mas esto debe ser objeto de tratamiento en otro estudio. Del presente debe extraerse la idea de que resulta indispensable la clarificación de situaciones, posiciones, definiciones y ámbitos, antes de abordar una regulación ordenada de las pensiones y de la Acción Complementaria de la Seguridad Social, si se quiere avanzar ordenadamente en un campo, que, cada día, resultará de mayor transcendencia para el ciudadano individual, la vida de la sociedad y la estabilidad económica.

\section{Bibliografía}

Almansa Pastor: Derecho de la Seguridad Social. Madrid.

Alonso García, R.: Derecho Comunitario. Ed. Ceura. Madrid.

Alonso Olea, M.: Aspectos sociales de la Comunidad Económica Europea. Anales de la Real Academia de Ciencias Morales y Políticas. Madrid.

Alonso Olea y TORTUERo: Instituciones de Seguridad Social. Madrid.

Alonso Olea, M.: Derecho del Trabajo. Editorial: Servicio Publicaciones Facultad de Derecho. Universidad Complutense de Madrid. Madrid.

Alonso Olea, M. y BARreiro,G.: Comentarios al Estatuto de los Trabajadores. Editorial Civitas. Madrid.

Borrajo Dacruz, E.: De las Comunidades Europeas a la Unión Europea: El acta única y la Europa Social. Actualidad Laboral n. 20.

Colina Robledo, M.: «La política social de las Comunidades Europeas». Revista de Trabajo n. $^{\circ} 78$.

Colina Robledo, M.: Derecho Social Comunitario. Tirant lo Blanch. Valencia. 
Colina Robledo, M.: «Relaciones laborales y condiciones de trabajo en el derecho comunitario europeo». La Política Social en la C.E. Madrid.

Colina Robledo, M.: «Aspectos sociales de la Presidencia española en el Consejo de las C.E.». Documentación laboral núms. 27/28.

COMISIÓn EUROPEA. «Informe sobre el estudio comparativo de las normas relativas y las condiciones laborales en los Estados miembros». Documentación Laboral, núms. 27/28.

Diego Cano: Políticas de privatización. Aproximación Teórica. Consejo Económico y Social. Madrid.

FERnÁndez LóPEZ, M. ${ }^{\text {a }}$ F.: Las Directivas laborales de la Comunidad Europea. Información a los trabajadores sobre sus condiciones de trabajo. II jornadas sobre la Comunidad Europea. Sevilla.

Fraga IrIBARnE, M.: «La dimensión social de la Europa Comunitaria». Revista Española de Derecho del Trabajo, n. 37.

FonT Galan, Juan Ignacio: La libre competencia en la Comunidad Europea, Publicaciones del Real Colegio de España, Bolonia.

Galiana Moreno, J.M.: «El lento camino de gestación de la política comunitaria». Revista Española de Derecho del Trabajo n. 37.

GÁRATE CASTRO: La racionalización de las pensiones de Seguridad Social. Pamplona.

García Becedas, G.: Introducción del Derecho Español del Trabajo. Madrid.

GuY, Isaac: Manual de Derecho Comunitario General.

JAVILliER, J.: Derecho del Trabajo. Editorial: Instituto de Estudios Laborales y de la Seguridad Social. Madrid.

LOSADA GonZÁleZ, Herminio: «Tratado de la UE». Textos consolidados de los Tratados Comunitarios. Secretaría de Estado para las Comunidades Europeas. Madrid.

LouIs, Jean Víctor: El ordenamiento jurídico comunitario, Colección. «Perspectivas Europeas». Bruselas.

Lluis y NaVAs, J.: Manual de Derecho Laboral. Barcelona.

Mangas Martín, Araceli: Derecho comunitario europeo y Derecho español. Editorial Extecom, S.A.

Ministerio DE TRABAJO: Informe de la Ponencia para el análisis de los problemas estructurales del sistema de la Seguridad Social y de las principales reformas que deberán acometerse.

Molina del Pozo, D.F.: Manual de Derecho de la Comunidad Europea. Madrid.

Montoya Melgar, A.: Armonización de políticas y derechos sociales. Instituciones de Derecho Social Europeo. Ed. Tecnos. Madrid.

Montoya Melgar, A. y otros: Comentarios al Estatuto de los Trabajadores. Editorial Aranzadi. Madrid.

Montoya Melgar, A.: Derecho del Trabajo. A. Montoya. Editorial: Tecnos. Madrid.

OlietPala, B.: La extensión de la responsabilidad en materia de Seguridad Social. Ministerio de Trabajo.

OlietPala, B.: Normas presupuestarias, pensiones públicas y protección social complementaria. Ministerio de Trabajo. 
Palomeque, C.: Derecho Sindical Español. Editorial: Tecnos. Madrid.

PÉrez AMORós, F.: «El derecho social de la Comunidad Europea y su incidencia en el ordenamiento laboral especial». Revista Técnico-Laboral. Vol. XI. n. ${ }^{\circ} 42$.

PÉREZ DE los Cobos, F.: Derecho Social comunitario en el Tratado de la Unión Europea. Ed. Civitas. Madrid.

Rey GuAnter, S.: «Los instrumentos normativos del Tratado de Roma en el desarrollo de la política social comunitaria». Relaciones Laborales n. ${ }^{\circ} 21$.

Rey Guanter, S.: Principios y tendencias en el Derecho Social Comunitario. II Jornadas sobre Comunidad Europea. Sevilla.

Rivas, Jonczy y SECHE: Derecho Social Europeo. Instituto de Estudios Laborales. Madrid.

RodríGuez RAmos, D.J. y otros: Sistema de Seguridad Social y otros. Madrid.

Roussomano, V.: Principios Generales de Derecho Sindical. Editorial: Instituto de Estudios Políticos, página 216. Madrid.

Sala Franco, T. y Ramírez Martínez, J.M. (1986): Introducción al Derecho Social Comunitario. Ed. Tirant lo Blanch. Valencia.

SERRANO DE TRIANA, A.: La responsabilidad subsidiaria en la Seguridad Social. Ministerio de Trabajo.

SuÁrez González, F.: El Espacio Social Europeo. Estudios ofrecidos al Prof. Alonso Olea. Madrid.

VILLA, L.E. y otros: Instituciones de Derecho del Trabajo. Editorial: Centro de Estudios Universitarios Ramón Areces. Madrid.

Wolfrang Daubler: Derecho del Trabajo. Editorial: Ministerio de Trabajo y Seguridad Social. Madrid. 